\title{
POPULATION STRUCTURE OF ANISAKIS SIMPLEX (NEMATODA) IN HARBOR PORPOISES PHOCOENA PHOCOENA OFF DENMARK
}

\author{
M. V. Herreras, J. A. Balbuena*, F. J. Aznar, S. E. Kaarstad, M. Fernández, and J. A. Raga \\ Marine Zoology Unit, Cavanilles Institute of Biodiversity and Evolutionary Biology, University of Valencia, Official P.O. Box 22085, 46071 \\ Valencia, Spain.e-mail: j.a.balbuena@uv.es
}

\begin{abstract}
The population structure and habitat selection of Anisakis simplex in 35 harbor porpoises off Denmark are described. The nematodes were collected from the stomach and duodenal ampulla and were categorized as third-stage larvae, fourth-stage larvae, subadults, and adults. The porpoises harbored 8,043 specimens of A. simplex. The proportion of adults and subadults increased with infrapopulation size. The number of development stages across infrapopulations covaried significantly (Kendall's test of concordance). Concordance was higher in hosts with the highest intensities than in those with low and medium intensities. All stages occurred mainly in the forestomach, but this trend was stronger for the adults. Adult and subadult sex ratios did not depart significantly from 1:1. Our data suggested that recruitment and duration of each stage were the main factors accounting for infrapopulation structure. The preference of A. simplex for the forestomach conformed with previous studies, but the narrower distribution of adults relative to other stages might indicate a strategy to enhance mating opportunities. Information on sex ratios of A. simplex is scarce and contradictory. We suggest that the discrepancies might partly reflect differences in categorization criteria and statistical methods.
\end{abstract}

The occurrence of third-stage larvae (L3) of anisakid nematodes in the fillets of commercial fish entails well-known public health concerns and economic repercussions (Burt, 1994; Smith, 1999). Consequently, the population ecology of anisakid nematodes has received more attention than that of any other parasite of marine mammals. Most population studies of anisakids have focused on describing the population structure and dynamics of the sealworm, Pseudoterranova decipiens, and other species common in seals (see Bowen 1990; Desportes and McClelland 2001, and references therein for examples) and have produced valuable data on the life history and population ecology of these nematode species.

As for the whaleworm, Anisakis simplex, most population studies have focused on its intermediate and paratenic hosts (Brattey and Bishop, 1992; Herreras et al., 2000; Podolska and Harbowy, 2003), whereas its population biology in the definitive hosts has been less studied. Most information on the definitive hosts concerns the harbor porpoise, Phocoena phocoena, a small odontocete common in the Northern Hemisphere (Smith, 1989; Brattey and Stenson, 1995; Herreras et al., 1997). The data on the population structure of A. simplex in this cetacean are based on small sample sizes (Young, 1972; Smith, 1989) or lack details of the infrapopulation structure (Lick, 1991; Brattey and Stenson, 1995). These 2 shortcomings are also common in studies of this nematode in other cetacean species (e.g., Wazura et al., 1986; Raga and Balbuena, 1993). Moreover, little is known about habitat selection by A. simplex in the definitive hosts. To our knowledge, there are only 2 studies providing such information (Brattey and Stenson, 1995; Aznar et al., 2003), and only the latter uses data at the infrapopulation level. Aznar et al. (2003) studied 4 sympatric cetacean species, and except for the franciscana Pontoporia blainvillei, the sample sizes of the other species were rather small.

Although knowledge of the infrapopulation structure of $A$. simplex in definitive hosts is scarce, it is at the infrapopulation level where useful information about the reproductive portion of the population can be found. In addition, infrapopulation data are crucial to understand the population ecology of parasites

Received 12 July 2003; revised 8 October 2003; accepted 8 October 2003.

* To whom correspondence should be addressed. because parasites have usually highly aggregated distributions. Thus, patterns arising from the pooled data of the component population might be biased at low sample sizes because they might miss the uncommon heavily infested hosts. Consequently, the objective of the present study was to describe the maturity structure and habitat selection of A. simplex at infrapopulation and component population levels. We expect that the patterns revealed by the present study will provide evidence for potential mechanisms determining population structure and habitat selection of $A$. simplex in the harbor porpoise. To our knowledge, this is the first study providing infrapopulation details of $A$. simplex in a definitive host based on a relatively large sample of cetaceans.

\section{MATERIALS AND METHODS}

\section{Data collection}

The present study describes the population structure of A. simplex found in 35 of 78 harbor porpoises surveyed for parasites. The porpoises were found stranded or were caught as by-catch in fishing gear between 1988 and 1990 in Danish waters $\left(54^{\circ} 33^{\prime}-57^{\circ} 45^{\prime} \mathrm{N}, 8^{\circ} 5^{\prime}-12^{\circ} 47^{\prime} \mathrm{E}\right)$ and were frozen within $24 \mathrm{hr}$ of detection. These animals were collected in accordance with applicable Danish legislation.

The stomach and duodenal ampulla (a funnel-shaped chamber at the proximal duodenum) were removed from frozen porpoises and examined for parasites. The nematodes were collected from the walls of the stomach and duodenal ampulla (sometimes attached to ulcers) or separated from food items using a $0.6-\mathrm{mm}$-mesh sieve. The stomach of the harbor porpoise consists of 3 stomach chambers: the forestomach, the main stomach, and the pyloric stomach (see fig. 2 in Gaskin [1978] for anatomical details). In 23 of the 35 porpoises, the 3 stomach chambers and the duodenal ampulla were surveyed independently for habitat selection analyses of third-stage larvae (L3), fourth-stage larvae (L4), subadults, and adults. The specimens were preserved in $70 \%$ ethanol and cleared in lactophenol for examination and identification. All the L3 collected corresponded to type I larvae (sensu Berland, 1961). Because A. simplex represents a complex of sibling species, we assumed that our specimens belonged to A. simplex sensu stricto as it is the only species of the complex recorded in the study area (Mattiucci et al., 1997). No other anisakid, except Hysterothylacium aduncum, occurred in the porpoises. Because the latter uses fish as definitive hosts, individuals of $A$. simplex sensu stricto formed the only viable population of anisakid nematodes in our sample of harbor porpoises.

The worms were counted and categorized as L3, L4, subadult female, subadult male, adult female, and adult male. Subadults and adults were distinguished from the larvae by the presence of a developing or fully developed reproductive system. Adult females were distinguished from the subadult females by the presence of eggs in the uterus and the adult 
males from the subadult males by the presence of sperm in the semina vesicle and distal part of the spermiduct and the fully developed spicules. The term "sexable" is used herein to refer to subadult and adult individuals globally. Voucher specimens of each development stage are deposited at the Natural History Museum in London (reg. nos 2003.8.20.1-28). The nematodes of each stage were counted in all porpoises except in 1 where over 3,000 individuals occurred. In this case, the few specimens occurring in the main and pyloric stomach ( 9 and 4 individuals, respectively) were counted and categorized to development stage, whereas the numbers of each stage located in the forestomach were estimated from a random subsample representing about one-fourth of the sample's total wet weight.

Some damaged specimens could not be allocated to any maturity stage, and these uncategorized nematodes were omitted in the statistica analyses. We assumed that their omission caused no bias in the patterns described because such worms did not seem to belong to any specific category. Eventually, 34 of the 35 infected porpoises were used for analysis of population structure composition because 1 animal was infected with an unclassified worm.

\section{Maturity structure}

The maturity structure of $A$. simplex was described at 2 levels: the component population, i.e., the pooled sample across hosts, and the infrapopulation, i.e., within the individual host (Bush et al., 1997).

Because the analysis of maturity patterns can provide information about potential mechanisms determining population structure (Aznar et al., 1997), we searched for significant patterns in our data in 2 ways. First, we used a Kendall's test of concordance to determine whether the relative proportions of development stages (L3, L4, subadult, and adult) covaried significantly in each infrapopulation. The effect of infrapopulation size on the concordance of development stages was assessed by performing the same test with 3 subsets of the sample consisting of 12 11 , and 11 individuals with low (1-3 worms), medium (4-129), and high $(139-3,244)$ intensities, respectively. Second, to establish whether the number of development stages covaried consistently across infrapopulations, we performed a Kendall's test of concordance on the transposed data matrix of development stages. The covariation in numbers of each maturity stage with infrapopulation size was tested with Spearman's correlation tests.

\section{Habitat selection}

We assumed that postmortem movements of worms did not significantly affect patterns of habitat selection because the digestive chambers are large compared with parasite size. The distribution of A. simplex across digestive chambers was described by calculating the percentage of L3s, L4s, subadults, and adults in each digestive compartment, both at component population and infrapopulation levels. In addition, we scored each worm according to its site of infection by assigning 1,2 , 3 , and 4 to the location in the fore-, main, and pyloric stomach and duodenal ampulla, respectively (Moore and Simberloff, 1990), to establish the location of the mean, anterior, and posterior worm of each development stage in each infrapopulation. To determine whether the distribution range of each development stage in the stomach expanded with increased infrapopulation size, we carried out Spearman's correlation tests relating habitat range of each development stage, i.e., location of posterior worm minus location of anterior worm, with total intensity.

\section{Sex ratios}

Because differences in sex ratio estimates might result from differences in the criteria used to estimate sex ratios (apparently some authors consider adult nematodes only, whereas others include all sexable worms in sex ratio estimation) (Brattey and Stenson, 1993), we estimated the sex ratio of subadult, adult, and sexable worms of the component population and infrapopulations. The sex ratio was expressed as the proportion of males (PM) of a particular stage (subadult, adult, and sexable) of the total number of worms of the corresponding stage. Because the PM distributions departed from normality, 95\% confidence intervals and median values of PM were calculated by nonparametric bootstrap using the bias-corrected and accelerated percentile algorithm (Efron and Tibshirani, 1993).

Chi-square tests (or $G$-tests) commonly used for statistical inference on sex ratio estimates might not be appropriate with parasite data because parasites are recruited in clusters from highly aggregated distributions in the intermediate or paratenic hosts. Recruitment in clusters violates the assumption of independence among observations (Kramer and Schmidhammer, 1992; Garson and Moser, 1995), and sampling from aggregated distributions usually results in increased type I error when the chi-square test is computed (Garson and Moser, 1995). It is out of the scope of this article to evaluate the magnitude of this error in parasite sex ratio inferences, but we used Wilcoxon signed rank tests for paired samples because the assumption of independence concern only the pairwise differences between observations, which is met by our data (Conover, 1999).

These tests were used to test whether the number of males and females in the subadult, adult, and sexable categories differed significantly from 1:1 across infrapopulations. Likewise, we established whether the PM estimates of subadult and adult worms differed significantly across infrapopulations with Wilcoxon signed rank tests using 15 of 20 hosts with sexable worms because 2 and 3 porpoises harbored only adult and subadult nematodes, respectively. The effect of infrapopulation size on the PM estimates was assessed by computing Spearman's correlation coefficient between infrapopulation PMs of subadult, adult, and sexable A. simplex and the corresponding infrapopulation size of each development category.

\section{Statistical criteria and terminology}

Statistical significance was set at the 0.05 probability level. Ecological terms are used as defined by Bush et al. (1997), but we also apply the term intensity to specific subsets of the sample defined by development categories, e.g., intensity of adult worms. Bootstrap calculations were performed with the bootstrap package (Efron and Tibshirani, 1993) under R 1.5.1 (Ihaka and Gentleman, 1996).

\section{RESULTS}

The 35 infected porpoises harbored 8,043 specimens of $A$. simplex (mean intensity \pm SD: $229 \pm 577$, range: 1-3,245), of which $514(6.4 \%)$ could not be allocated to any development category. Both at infrapopulation and component population levels, L3s and subadults accounted for the lowest proportions, whereas adult worms and L4s accounted for the largest share of the population (Table I). The proportion of development stages changed with infrapopulation size, i.e., the proportions of $\mathrm{L} 3 \mathrm{~s}$ and L4s were higher at low intensities, whereas those of adults and subadults increased at high intensities (Fig. 1). The percentage of sexable nematodes was significantly correlated with infrapopulation size $\left(r_{\mathrm{s}}=0.893, P=0.001\right)$. The composition of development stages across infrapopulations was significantly concordant $(W=0.255, P<0.001)$, but this concordance was much higher in the host subset showing the highest intensities of $A$. simplex $(W=0.675, P<0.001)$ than in those having low and medium intensities $(W=0.252, P<$ 0.023 and $W=0.252, P<0.035$, respectively). The covariation in intensity of the development stages across infrapopulations was highly significant $(W=0.790, P<0.001)$, and the intensity of each stage increased strongly with total infrapopulation size $\left(r_{\mathrm{s}}\right.$ ranged between 0.76 and 0.93 and $P<0.001$ in all 4 correlations)

The 23 hosts used for habitat selection analyses harbored 6,597 worms. Twenty-two hosts had worms in the forestomach, 7 in the main stomach, 6 in the pyloric stomach, and 2 in the duodenal ampulla. In 15 of the 23 porpoises, worms were confined to 1 chamber exclusively, whereas 5 hosts harbored worms in the fore-, main, and pyloric stomach, 2 hosts had nematodes in the fore- and main stomach, and 1 host harbored worms in the fore- and pyloric stomach simultaneously.

All developmental categories were mostly confined to the 
TABLE I. Composition by development stage of the infrapopulations and component population of Anisakis simplex from 34 harbor porpoises off Denmark.

\begin{tabular}{|c|c|c|c|c|}
\hline Development stage & \multicolumn{2}{|c|}{ Infrapopulation size } & $\begin{array}{c}\% \text { Infrapopulation } \\
(\bar{x} \pm \mathrm{SD})\end{array}$ & $\begin{array}{c}\text { Component } \\
\text { population }(\%)\end{array}$ \\
\hline L3 & $31.1 \pm 97.9$ & 1 & $23.5 \pm 3.4$ & $1,057(14.0)$ \\
\hline Larvae (L3 + L4) & $103.7 \pm 255.0$ & 13 & $68.5 \pm 3.6$ & $3,524(46.8)$ \\
\hline Subadult male & $9.7 \pm 23.6$ & 0.5 & $3.7 \pm 6.5$ & $331(4.4)$ \\
\hline Subadult female & $9.6 \pm 20.7$ & 0 & $4.6 \pm 1.6$ & $326(4.3)$ \\
\hline Adult female & $51.4 \pm 156.2$ & 1 & $12.7 \pm 19.9$ & $1,747(23.2)$ \\
\hline Adult (total) & $98.5 \pm 288.6$ & 2 & $23.2 \pm 28.5$ & $3,348(44.5)$ \\
\hline Sexable (total) & $117.8 \pm 329.8$ & 4.5 & $31.5 \pm 33.6$ & $4,005(53.2)$ \\
\hline
\end{tabular}

forestomach (Table II), but this trend was stronger for the adult nematodes, where over $99.8 \%$ of the component population and $99.9 \%$ of the infrapopulation average occurred in this chamber. Larval stages showed broader distribution ranges (higher SDs) and occurred in all the digestive chambers, whereas subadults and adults had narrower distributions and did not occur in the duodenal ampulla (Table II). The analysis of the mean ranking position of the anterior, posterior, and median worm of each development stage showed that the average median worm was posteriorly ordered according to development sequence (adults, subadults, L4s, and L3s), and the larval stages showed wider distribution ranges than the sexable worms (Fig. 2). None of the distribution range of development stages expanded significantly with infrapopulation size (1-tailed $r_{\mathrm{s}}<0.47, P>0.05$ in all 4 correlations).

Table III shows the estimated PMs for subadult, adult, and sexable worms. The number of males and females across infrapopulations did not differ significantly from 1:1 in any of the development categories (subadults, adults, and sexables) (Wil-

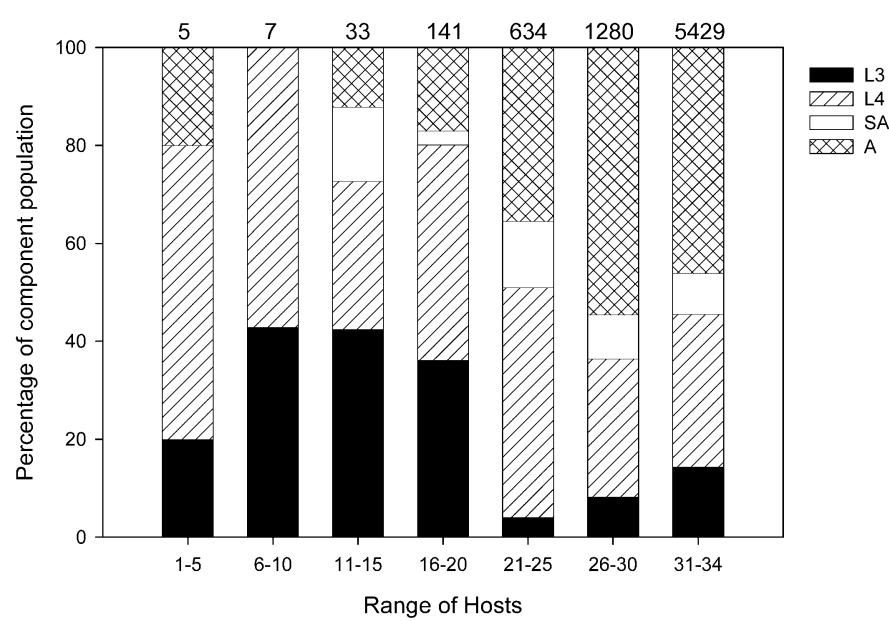

FIGURE 1. Variation in the proportion of component population development stages of Anisakis simplex with infrapopulation size in 34 harbor porpoises. Hosts were ranked from 1 to 34 and categorized according to infrapopulation size of A. simplex. Numbers on top of bars indicate the number of worms in each category. Development stages of A. simplex are coded as follows: L3, third-stage larvae; L4, fourth-stage larvae; SA, subadults; A, adults. coxon signed rank tests: $P>0.60$ in all cases), and the PMs of subadult and adult worms did not differ significantly (Wilcoxon signed rank tests: $P>0.77$ ). Infrapopulation PMs of subadult, adult, and sexable worms were not significantly correlated with the infrapopulation size of the respective development stage $\left(r_{\mathrm{s}}<-0.15\right.$, and $P>0.60$ in all cases). The median estimates of the bootstrapped PMs for subadult, adult, and sexable worms were similar to their respective component population and mean infrapopulation PMs (Table III).

\section{DISCUSSION}

\section{Maturity structure}

Evidence from the present study suggests that the infrapopulation structure in a given porpoise is primarily the result of recruitment and development rates of each stage. All development stages covaried positively, and their relative proportion became more predictable with increased infrapopulation size. Assuming that larger infrapopulations are older and result from several infestation waves, this pattern might arise because the proportions of development stages would tend to adjust to their relative duration in time. Conversely, if small infrapopulations were the result of a single recruitment wave, worms would tend to be in the same stage (L3 for instance, if just ingested) and their structure would be more unpredictable. This scenario is supported by our study revealing lower concordance between development stages at low and medium infections (Kendall's tests). In addition, under a model of infection regulated by recruitment and development rates, increasing infrapopulation size leads to 2 predictions that are clearly supported by our data. First, all development stages should increase consistently with total intensity (Kendall's test across infrapopulations and Spearman's correlations with total intensity). Second, nongravid stages (subadults) should be rare at low intensities because of their transient condition (Fig. 1). Although there is no information from field experiments, both laboratory infections in rats (Gibson, 1970) and in vitro studies of A. simplex (Grabda, 1976; Iglesias et al., 2001) indicate that L3s, and subadults in particular, are short-lived in comparison with L4s and adults, which conforms with the compositions of the component population and average infrapopulation observed in the present study. 
TABLE II. Habitat distribution of Anisakis simplex in the stomach and duodenal ampulla of 23 harbor porpoises from Danish waters. Data are expressed as percentage of infrapopulation and component population collected from each digestive chamber.

\begin{tabular}{|c|c|c|c|c|c|c|c|c|}
\hline \multirow[b]{2}{*}{$\begin{array}{c}\text { Development } \\
\text { stage }\end{array}$} & \multicolumn{2}{|c|}{ Forestomach } & \multicolumn{2}{|c|}{ Main stomach } & \multicolumn{2}{|c|}{ Pyloric stomach } & \multicolumn{2}{|c|}{ Duodenal ampulla } \\
\hline & $\begin{array}{l}\text { Infrapopulation } \\
\quad(\bar{x} \pm \mathrm{SD})\end{array}$ & $\begin{array}{l}\text { Component } \\
\text { population }\end{array}$ & $\begin{array}{l}\text { Infrapopulation } \\
(\bar{x} \pm \mathrm{SD})\end{array}$ & $\begin{array}{l}\text { Component } \\
\text { population }\end{array}$ & $\begin{array}{l}\text { Infrapopulation } \\
\quad(\bar{x} \pm \mathrm{SD})\end{array}$ & $\begin{array}{l}\text { Component } \\
\text { population }\end{array}$ & $\begin{array}{l}\text { Infrapopulation } \\
\quad(\bar{x} \pm \mathrm{SD})\end{array}$ & $\begin{array}{c}\text { Component } \\
\text { population }\end{array}$ \\
\hline L3 & $79.5 \pm 39.9$ & 98.2 & $0.02 \pm 0.09$ & 0.21 & $20.1 \pm 40.0$ & 1.13 & $0.1 \pm 0.4$ & 0.51 \\
\hline L4 & $91.8 \pm 23.9$ & 95.1 & $3.5 \pm 11.2$ & 0.44 & $4.8 \pm 21.4$ & 4.39 & $0.03 \pm 0.15$ & 0.10 \\
\hline Subadult & $99.9 \pm 0.4$ & 99.5 & $0.07 \pm 0.25$ & 0.37 & $0.03 \pm 0.12$ & 0.18 & $0.00 \pm 0.00$ & 0.00 \\
\hline Adult & $99.97 \pm 0.10$ & 99.8 & $0.01 \pm 0.05$ & 0.10 & $0.01 \pm 0.05$ & 0.10 & $0.00 \pm 0.00$ & 0.00 \\
\hline Total & $88.0 \pm 28.3$ & 98.1 & $2.1 \pm 7.2$ & 0.24 & $9.9 \pm 25.8$ & 1.58 & $0.03 \pm 0.14$ & 0.11 \\
\hline
\end{tabular}

\section{Habitat selection}

The preference of A. simplex for the forestomach in general, and of adult worms in particular, observed in the present study conforms to previous evidence concerning A. simplex sensu lato, both in the harbor porpoise (Lick, 1991; Brattey and Stenson, 1995) and in other odontocetes (Aznar et al., 2003, and references therein). Aznar et al. (2003) showed that adults of A. simplex occurred mostly in the main stomach of franciscanas, whereas it appeared mainly in the forestomach of other 3 sympatric cetacean species. Because the franciscanas lack a forestomach, these authors concluded that adults of $A$. simplex seem to preferably inhabit the first stomach chamber of odontocetes. In addition, data from each of the 4 odontocetes species showed that whereas adults were mainly restricted to the first stomach chamber, the other development stages were ordered posteriorly in developmental sequence (Aznar et al., 2003). Although this pattern resulted from a small number of hosts having forestom-

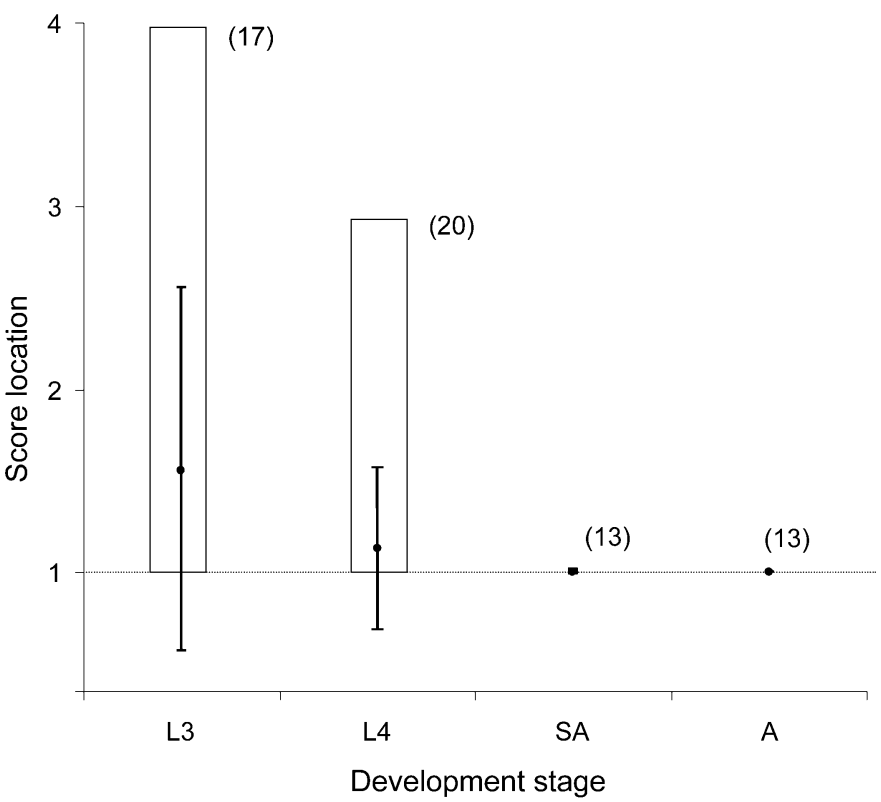

FIGURE 2. Position by development stages of the average (circles) and maximum and minimum (boxes) mean scores of Anisakis simplex from 23 harbor porpoises. Error bars represent \pm 1 SD of the average mean score. Scores of site of infection were assigned as follows: 1, forestomach; 2, main stomach; 3, pyloric stomach; and 4, duodenal ampulla. Numbers in parentheses indicate sample size for each development stage. ach (12 individuals in total), it is clearly confirmed by our data based on a larger sample size (Fig. 2).

However, because the preference of A. simplex for the first stomach chamber is not universal (Uchida et al., 1998), further information on the relative suitability of stomach chambers, the density dependence of $A$. simplex distributions, and the differences in habitat selection patterns between sibling A. simplex species is needed to understand the mechanisms accounting for the stomach distribution of A. simplex sensu lato.

In the odontocetes studied (Aznar et al., 2003; present study), the apparent preference of A. simplex for the first stomach chamber may be explained by a combination of gravity, taking the infesting L3s to the ventral hemisphere of the first chamber (Smith, 1989), and the conditions created where the first digestion occurs (Aznar et al., 2003). However, the greater restriction of adult A. simplex to the first stomach relative to the other development stages reflects perhaps a secondary adaptation to enhance mating opportunities. Because intraspecific competition did not seem to operate at least at the habitat scale considered (the distribution of development stages across the stomach chambers did not expand with intensity), the wider distribution ranges of developing stages compared with adult A. simplex might indicate either an anteriad ontogenetic migration or a posteriad displacement of inviable developing worms.

\section{Sex ratios}

Information on sex ratios of A. simplex is very scarce. Based on the raw data reported by Young (1972) and Smith (1989), the overall PMs of A. simplex in harbor porpoises off Scotland are 0.48 and 0.49 , respectively. Although the number of porpoises harboring sexable worms was very small in both studies ( 5 and 3 , respectively), these sex ratios are very similar to our

TABLE III. Sex ratio estimates of Anisakis simplex expressed as PM of the total population of each development category: subadults, adults, and sexables.

\begin{tabular}{lcccccc}
\hline $\begin{array}{c}\text { Develop- } \\
\text { ment } \\
\text { stage }\end{array}$ & $\begin{array}{c}\text { Component } \\
\text { population }\end{array}$ & $\begin{array}{c}\text { Infrapopulation } \\
(\bar{x} \pm \text { SD })\end{array}$ & & & \multicolumn{2}{c}{ Bootstrap } \\
\cline { 5 - 7 } \cline { 5 - 7 } & Subadult & 0.504 & $0.512 \pm 0.230$ & 17 & 0.506 & $0.432-0.568$ \\
Adult & 0.478 & $0.470 \pm 0.194$ & 18 & 0.478 & $0.456-0.511$ \\
Sexable & 0.482 & $0.497 \pm 0.191$ & 20 & 0.482 & $0.459-0.513$ \\
\hline
\end{tabular}

* $95 \%$ confidence intervals calculated by the bias-corrected and accelerated percentile method (Efron and Tibshirani, 1993). 
PM estimates. In contrast, Brattey and Stenson's (1995) data on harbor porpoises off Newfoundland and Labrador suggest a PM of sexable A. simplex of 0.29. A similar disagreement in sex ratio estimates between studies has also been observed in several anisakid species from seals. For a given species, some authors report a bias toward females, whereas others report sex ratios of about 1:1 (Brattey and Stenson, 1993, and references therein)

Brattey and Stenson (1993) proposed for anisakids of seals that such discrepancies might partly result from differences in the criteria used to estimate sex ratios, considering all sexable worms or adult worms only. In the former case, sex ratios would tend to be biased toward females, whereas they would be approximately even in the latter. Evidence from our study did not conform to this expectation because the difference between sex ratios of adult and sexable worms was not significant and because both sex ratios were close to $1: 1$. This result adds to the contradictory evidence of anisakid sex ratios accumulated in the literature, but we believe that the confused situation can be remedied by setting standards for criteria to estimate sex ratios and for statistical inference.

Differences in mortality rates between the sexes have been proposed as a factor contributing to heterogeneities in anisakid sex ratios between hosts (Brattey and Ni, 1992; Brattey and Stenson, 1993). Note also that differences in maturity rates between parasite genders can also contribute to sex ratio heterogeneity between infrapopulations. Although we cannot rule out the intervention of differential mortality (or maturity) rates between sexes of $A$. simplex, our results do not provide evidence for any factor other than chance accounting for sex ratio differences between porpoises. The sex ratios of subadult and adult worms were close to $1: 1$ and did not differ significantly from each other. In addition, when a higher mortality in 1 of the genders occurs, a monotonic increase or decrease in sex ratios with intensity of infection is often observed (Helle and Valtonen, 1980). However, in the present study, PMs were not correlated with infrapopulation size.

By focusing on the infrapopulation structure of A. simplex, our study provides new data on the population ecology of this nematode in the definitive host. The present study suggests that it is difficult to draw conclusions about the population ecology of A. simplex and other anisakids partly because current evidence is based on observational data, often on small sample sizes, and also because of differences in categorization criteria of development stages and in statistical methods for data analysis. We hope that this article serves as a plea for consensus on the methodology to address these issues.

\section{ACKNOWLEDGMENTS}

We thank Carl Kinze, Zoological Museum of Copenhagen, for collecting the porpoises for this study within a research project supported by the World Wildlife Fund and the Danish Federation for Animal Protection. Al Bush, Department of Zoology, Brandon University, gave us valuable advice and suggestions for data analysis. Al Shostak and 2 anonymous referees provided valuable criticism and comments. This study was funded by the DGICYT of the Spanish Government (Project PB92-875). F.J.A. and M.F. hold Ramón y Cajal Research Fellowships from the Ministry of Science and Technology of Spain.

\section{LITERATURE CITED}

Aznar, F. J., J. A. Balbuena, A. O. Bush, and J. A. Raga. 1997. Ontogenetic habitat selection by Hadwenius pontoporiae (Digenea:
Campulidae) in the intestine of franciscanas (Cetacea). Journal of Parasitology 83: 13-18.

, M. V. Herreras, J. A. Balbuena, and J. A. Raga. 2003. Population structure and habitat selection by Anisakis simplex in four odontocete species from northern Argentina. Comparative Parasitology 70: 66-71.

Berland, B. 1961. Nematodes from some Norwegian marine fishes. Sarsia 2: $1-50$.

Bowen, W. D. 1990. Population biology of sealworm (Pseudoterranova decipiens) in relation to its intermediate and seal hosts. Canadian Bulletin of Fisheries and Aquatic Sciences 222: 306 p.

Brattey, J., AND C. A. Bishop. 1992. Larval Anisakis simplex (Nematoda: Ascaridoidea) infection in the musculature of Atlantic cod, Gadus morhua, from Newfoundland and Labrador. Canadian Journal of Fisheries and Aquatic Sciences 49: 2635-2647.

- AND I.-H. NI. 1992. Ascaroid nematodes from the stomach of harp seal, Phoca groenlandica, from Newfoundland and Labrador. Canadian Journal of Fisheries and Aquatic Sciences 49: 956-966.

- AND G. B. STENSON. 1993. Host specificity and abundance of parasitic nematodes (Ascaridoidea) from the stomachs of five phocid species from Newfoundland and Labrador. Canadian Journal of Zoology 71: 2156-2166.

, AND - 1995. Helminth parasites of the alimentary tract of the harbor porpoise, Phocoena phocoena (L.), from Newfoundland and Labrador. Journal of the Helminthological Society of Washington 62: 209-216

BurT, M. D. B. 1994. The sealworm situation. In Parasitic diseases: Epidemiology and ecology, M. E. Scott, and G. Smith (eds.). Academic Press, San Diego, California, p. 347-362.

Bush, A. O., K. D. Lafferty, J. M. Lotz, and A. W. Shostak. 1997. Parasitology meets ecology on its own terms: Margolis et al. revisited. Journal of Parasitology 83: 575-583.

Conover, W. J. 1999. Practical nonparametric statistics, 3rd ed. John Wiley \& Sons, New York, 584 p.

Desportes, G., AND G. McClelland. 2001. Sealworm in the North Atlantic: Ecology and population dynamics, vol. 3. NAMMCO Scientific Publications, Troms $\varnothing$, Norway, $171 \mathrm{p}$.

EFRON, B., AND J. R. TIBSHIRANI. 1993. An introduction to the bootstrap. Chapman \& Hall, New York, 436 p.

Garson, G. I., AND E. B. Moser. 1995. Aggregation and the Pearson chi-square statistic for homogeneous proportions and distribution in ecology. Ecology 76: 2258-2269.

GASKIN, D. E. 1978. Form and function in the digestive tract and associated organs in Cetacea, with a consideration of metabolic rates and specific energy budgets. Oceanography and Marine Biology an Annual Review 16: 313-345.

GiBson, D. I. 1970. Aspects of the development of "herringworm" (Anisakis sp. larva) in experimentally infected rats. Nytt Magasin for Zoologi 18: 175-187.

GRABDA, J. 1976. Studies on the life cycle and morphogenesis of Anisakis simplex Rudolphi, 1809 (Nematoda: Anisakidae) cultured in vitro. Acta Ichthyologica et Piscatoria 6: 119-141.

Helle, E., AND T. VAltonen. 1980. On the occurrence of Corynosoma spp. (Acanthocephala) in ringed seals (Pusa hispida) in the Bothnian Bay, Finland. Canadian Journal of Zoology 58: 298-303.

Herreras, M. V., F. J. Aznar, J. A. Balbuena, and J. A. Raga. 2000. Anisakid larvae in the musculature of the Argentinean hake, Merluccius hubbsi. Journal of Food Protection 63: 1141-1143.

, S. E. Kaarstad, J. A. Balbuena, C. C. Kinze, and J. A. Raga. 1997. Helminth parasites of the digestive tract of the harbour porpoise Phocoena phocoena in Danish waters: A comparative geographical analysis. Diseases of Aquatic Organisms 28: 163-167.

Iglesias, L. A., A. Valero, R. Benítez, and F. J. Adroher. 2001. In vitro cultivation of Anisakis simplex: Pepsin increases survival and moulting from fourth larval to adult stage. Parasitology 123: 285291.

IhaKa, R., And R. Gentleman. 1996. R: A language for data analysis and graphics. Journal of Computational and Graphical Statistics 5: 299-314.

Kramer, M., And J. Schmidhammer. 1992. The chi-square statistic in ethology: Use and misuse. Animal Behavior 44: 833-841.

Lick, R. R. 1991. Untersuchungen zu Lebenszyklus (Krebse-Fischemarine Säuger) und Gefrierresistenz anisakider Nematoden in 
Nord- und Ostsee. Berichte aus dem Institut für Meereskunde an der Christian-Albrechts-Universität, Nr. 128, Kiel, Germany, 195 p.

Mattiucci, S., G. Nascetti, R. Cianchi, L. Paggi, P. Arduino, L. Margolis, J. Brattey, S. Webb, S. D’Amelio, P. Orecchia, and L. Bullini. 1997. Genetic and ecological data on the Anisakis simplex complex, with evidence for a new species (Nematoda, Ascaridoidea, Anisakidae). Journal of Parasitology 83: 401-416.

Moore, J., And D. Simberloff. 1990. Gastrointestinal helminth communities of bobwhite quail. Ecology 71: 344-359.

Podolska, M., AND J. HARBOwy. 2003. Infection of Baltic herring ( $\mathrm{Clu}$ pea harengus membras) with Anisakis simplex larvae, 1992-1999: A statistical analysis using generalized linear models. ICES Journal of Marine Science 60: 85-93.

Raga, J. A., And J. A. Balbuena. 1993. Parasites of the long-finned pilot whale, Globicephala melas (Traill, 1809), in European waters. In Biology of Northern Hemisphere pilot whales, G. P. Donovan, C. H. Lockyer, and A. R. Martin (eds.). International Whaling Commission, Cambridge, U.K., p. 391-406.
Smith, J. W. 1989. Ulcers associated with larval Anisakis simplex B (Nematoda: Ascaridoidea) in the forestomach of harbour porpoises Phocoena phocoena (L.) Canadian Journal of Zoology 67: 22702276.

1999. Ascaridoid nematodes and pathology of the alimentary tract and its associated organs in vertebrates, including man: A literature review. Helminthological Abstracts 68: 49-96.

Uchida, A., Y. Kawakami, S. YuZu, S. Kishikawa, T. Kuramochi, J. AraKi, M. MachidA, AND K. NAgASAWA. 1998. Prevalence of parasites and histopathology of parasitation in minke whales (Balaenoptera acutorostrata) from the western North Pacific Ocean and the southern Sea of Okhotsk. Reports of the International Whaling Commission 48: 475-479.

Wazura, K. W., J. T. Strong, C. L. Glenn, and A. O. Bush. 1986. Helminths of the beluga whale (Delphinapterus leucas) from the Mackenzie River delta, Northwest Territories. Journal of Wildlife Diseases 22: 440-442.

Young, P. C. 1972. The relationship between the presence of larval anisakine nematodes in cod and marine mammals in British home waters. Journal of Applied Ecology 9: 459-483. 